\title{
Overlapping Deficiencies Refine the Map Position of the Sex-Linked Actin Gene of Drosophila melanogaster
}

\author{
Ann Sodja ${ }^{1}$, Rose M. Rizki ${ }^{2}$, T.M. Rizki ${ }^{2}$, and Rasheeda S. Zafar ${ }^{1}$ \\ 1 Department of Biological Sciences, Wayne State University, Detroit, Michigan 48202, U.S.A.; \\ 2 Division of Biological Sciences, University of Michigan, Ann Arbor, \\ Michigan 48109, U.S.A.
}

\begin{abstract}
A ${ }^{3} \mathrm{H}$-labelled actin-specific probe was hybridized to Drosophila melanogaster $\mathrm{X}$ chromosomes heterozygous for deficiencies in the $5 \mathrm{C}$ region. The results suggest that the sex-linked actin gene resides in the overlap region of $D f(1) C 149$ and $D f(1) N 73$ at $5 C 3-4$.
\end{abstract}

\section{Introduction}

How the morphology of a cell is molded as it occupies its final position in a developing tissue is a major question in understanding cell and tissue differentiation. There is now no doubt that cytoskeletal molecules such as tubulins and actins play important roles in this developmental phenomenon. In addition to maintaining cell structure and cytoskeleton, actin is involved in the contractile apparatus of muscle, cell motility, and cytokinesis. A family of closely related actin proteins appears to be involved in these processes and seems to function in a tissue specific manner (Huxley, 1969; Pollard and Weihing, 1974; Korn, 1978). Since the actins differ in their amino acid sequences, they must be coded for by separate genes (Vandekerckhove and Weber, $1978 \mathrm{a}, \mathrm{b}$ ). Actin genes from a number of different species have been cloned and characterized, and in a majority of the cases, it appears that the actins are coded by a multigene family. Depending on the species, the number of members in the family varies from only a few to about twenty.

In Drosophila, six actin genes have been found and localized to six disperse loci on the polytene chromosomes by in situ hybridization: $5 \mathrm{C}$ on the $\mathrm{X}$ chromosome, $42 \mathrm{~A}$ and $57 \mathrm{~A}$ on $2 \mathrm{R}, 79 \mathrm{~B}$ on $3 \mathrm{~L}$, and $87 \mathrm{E}$ and $88 \mathrm{~F}$ on 3R (Fyrberg et al., 1980, 1981). These genes are being characterized by molecular techniques and it would be worthwhile to combine genetic approaches with this accumulating biochemical information. To do so, it is important to know linkage relationships of the actin genes, and whether they are associated with any known morphological lesions. Map assignment of the actin genes by in situ hybridization was based on invariability of 
high grain density at the six regions since the probe used in the study gave higher backgrounds than probes for a number of other genes (Fyrberg et al., 1980). Furthermore, it is clear from Bridges and Bridges (1939) and Bridges' (1941) cytological maps of the salivary gland chromosomes that the number of bands in these regions varies from 3 in $79 \mathrm{~B}$ to 19 in $42 \mathrm{~A}$. A more precise localization of actin DNA sequences to the polytene chromosomes can be achieved by using overlapping deficiencies or other chromosomal aberrations where the breakpoints are known. We chose the $5 \mathrm{C}$ region for analysis since overlapping deficiencies in this region are available, and a number of mutant genes that affect wing morphogenesis such as curlex, crossveinless and deformed antennae are associated with bands spanning the region 5A8-5D6 (Lindsley and Grell, 1968). $5 \mathrm{C}$ is the locus for $\lambda \mathrm{DmA} 2$ which codes for a cytoplasmic actin (Fyrberg et al., 1980).

\section{Materials and Methods}

Three sex-linked deficiencies isolated by Dr. George Lefevre were obtained from the Drosophila Stock Center, California Institute of Technology. The $D f(1) H F 366$ chromosome lacks bands $3 \mathrm{E} 8$ through $5 \mathrm{~A} 7$ and is deficient for markers cho, ec, bi, peb, rb, rg, but complements bo and $c x$ mutants (for a description of symbols, see Lindsley and Grell, 1968). This deficiency does not extend into the $5 \mathrm{C}$ region but was selected to visualize hybridization at a $5 \mathrm{C}$ locus near a heterozygous deficient region. $D f(1) C 149$ extends from $5 \mathrm{~A} 10$ to $5 \mathrm{C} 4 \pm$; it is deficient for the $c v$ locus but not rux. The limits of the third deficiency used, $D f(1) N 73$, are defined by breakpoints at 5C3 and 5D5-6; it is deficient for rux but not $c v$ and $v s$. The deficiency (Df) chromosomes are maintained against an FM6 or FM7 balancer chromosome carrying yellow and Bar markers. Females heterozygous for each of the $D f$ chromosomes were crossed to yellow, chocolate males. Larvae from the crosses were raised on cream of wheat medium seeded with live yeast for $72 \mathrm{~h}$ at $27^{\circ} \mathrm{C}$. They were then transferred to an $18^{\circ} \mathrm{C}$ incubator for $12-18 \mathrm{~h}$ when the female larvae with black mouthparts were selected for chromosome squashes.

The actin coding sequence was isolated from a lambda-Drosophila DNA recombinant, $\lambda \mathrm{DmA} 2$ shown in Figure 1. The Sal I fragments indicated in the Figure were subcloned into pBR322, and the $0.8 \mathrm{~kb}$ fragment, representing the internal actin coding region only, was used to construct a radioactive probe. The plasmid containing the $0.8 \mathrm{~kb}$ fragment was cut with Sal I restriction endonuclease, separated on $0.7 \%$ agarose gel by electrophoresis and electroeluted from the gel (Sodja et al., 1982). The electroeluted fragment was labelled with tritium by nick translation (Maniatis et al., 1975). ${ }^{3} \mathrm{H}-\mathrm{dCTP}(107 \mathrm{Ci} / \mathrm{m} \mathrm{mol})$ was the radioactive nucleotide used. Specific activities of approximately $5 \times 10^{5} \mathrm{cpm} / \mu \mathrm{g}$ were obtained.

The preparation of the salivary gland chromosomes and subsequent hybridization with the ${ }^{3} \mathrm{H}$-actin probe was essentially according to the standard procedures (Gall and Pardue,

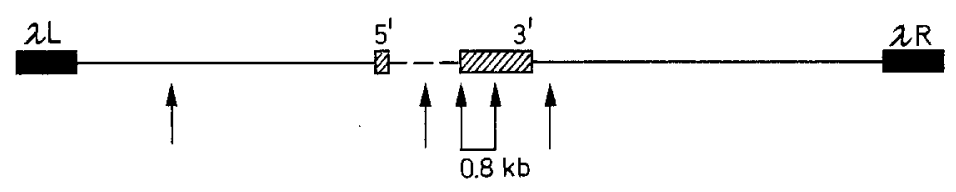

Fig. 1. A partial map of $\lambda \mathrm{DmA} 2$ with the Sal I restriction endonuclease sites indicated by arrows. The Dm DNA insert is between the $\lambda \mathrm{L}$ and $\lambda \mathrm{R}$ blocks, the actin coding sequence is indicated with barred blocks, and the intervening sequence with a dashed line. Transcriptional orientation of the actin gene is also noted. The Sal I fragments were subcloned into pBR322 and the $0.8 \mathrm{~kb}$ fragment prepared for nick translation and labelled with ${ }^{3} \mathrm{H}-\mathrm{dCTP}$ as described in Materials and Methods 
1971; Bonner and Pardue, 1970). We used a nick translated DNA probe to facilitate its handling and reduce the background. Hybridization was under siliconized coverslips using $10 \mu \mathrm{l}$ of $50 \%$ formamide ( $3 \times$ recrystallized), $0.5 \mathrm{M} \mathrm{NaCl}, 0.01 \mathrm{M}$ PIPES ( $\mathrm{pH}$ 7.2) containing $130 \mu \mathrm{g} / \mathrm{ml}$ each of yeast tRNA and calf thymus DNA. Approximately $300-500 \mathrm{cpm}$ were used per slide. The hybridization followed standard procedure, except that in the post-hybridization washes, the RNase step was omitted since the probe was nick translated DNA. Slides were developed and stained with Giemsa after 24 days of autoradiography.

\section{Results and Discussion}

DfHF366 is a relatively large deficiency distal to the $5 \mathrm{C}$ region. We selected this chromosome which is not deficient for $5 \mathrm{C}$ to serve as a control. Since both homologues of the HF366 heterozygous chromosome carry the 5C region, hybridization of the actin probe to these chromosomes should show silver grains over both of the paired homologues. Figure 2a shows the $D f^{+} /$ DfHF366 loop and the position of silver grains as expected to the right of the proximal breakpoint. We examined at least 20 such chromosomes where the silver grains were clearly distributed across both synapsed chromosomes. HF366 is a large deficiency so squashes of this region of the $\mathrm{X}$ chromosome generally spread flat and the $5 \mathrm{C}$ region with its overlying silver grains was displayed clearly. However, the $D f^{+} / D f H F 366$ larvae were not as healthy as larvae from the crosses with the two smaller deficiencies and their salivary gland chromosomes were small.

Examples of in situ hybridization in chromosomes heterozygous for $D f(1) C 149$ are given in Figure $2 \mathrm{~b}-\mathrm{d}$. Since the synapsed $D f^{+} / D f$ region is relatively small, the chromosomes were often twisted or bent in this region making analysis difficult. Ideal spreads where the $D f^{+}$region with the extra bands and coincident silver grains were found, however, such as in Figure $2 \mathrm{~b}, \mathrm{c}$. The preparation in Figure $2 \mathrm{~d}$ shows the pattern commonly seen where the $D f^{+}$region protrudes as a wedge of extra bands with silver grains localized on it. This pattern is quite distinct from that found in Figure $2 \mathrm{a}$. However, if the $D f$ in the squashed preparation comes to rest directly below the focal plane of the $D f^{+}$region, then the $D f^{+}$region appears lozengeshaped and the silver grains in this case are localized across the width of the chromosome. Thus, our interpretation of the hybridization site required evaluation of the position of the two synapsed homologues and the silver grains. In the $D f^{+} / D f C 149$ preparations we found 7 chromosomes where asynapsis extended from the telomere to the proximal region, or from the telomere to the middle section of the $\mathrm{X}$ chromosome. In all cases, only one of the homologues had silver grains in the $5 \mathrm{C}$ region. The absence of silver grains in the other homologue is consistent with the observations presented above, but we were reluctant to score these cases since the two arms were spatially separated in all cases.

Figure $2 \mathrm{e}$ shows an asynaptic region involving $D f(1) N 73$ where the asynapsis occurs over a short distance and the banding patterns of the two homologues can be clearly discerned. Silver grains are limited to the $D f^{+}$region of the asynapsed homologue where the extra bands are clearly visible. In addition to this preparation we found 2 asynapsed $D f^{+} / D f N 73$ 

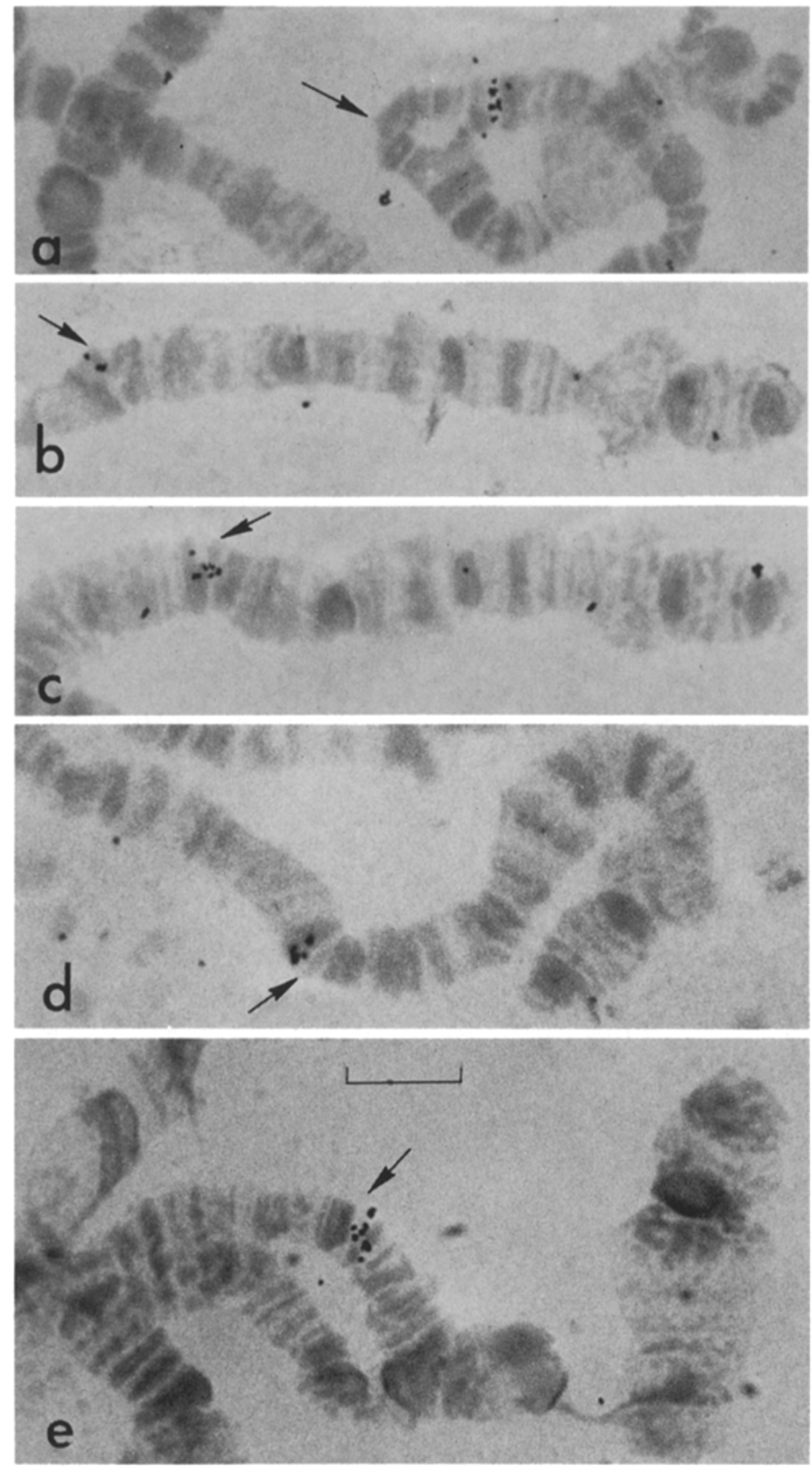

Fig. 2a-e. Autoradiographs of the distal ends of $X$ chromosomes following hybridization with a ${ }^{3} \mathrm{H}$-labelled actin coding sequence. The arrows point to the $D f^{4}$ strand in each case. Bar represents $10 \mu \mathrm{m}$. a Silver grains span the $5 \mathrm{C}$ region lying to the right of the large $D f^{+}$loop in a $D f^{+} / D f(1) H F 366$ chromosome. b-d $D f^{+} / D f(1) C 149$ chromosomes showing silver grains associated with the $D f^{+}$homologue. When the $D f^{+} / D f$ is squashed to clearly display the $D f^{+}$bands, the silver grains are localized to the right end of this region (b). e Asynapsed homologues of a $D f^{+} / D f(1) N 73$ chromosome with silver grains on the $D f^{+}$strand 
chromosomes similar to those described above for $D f C 149$. We also scored 20 preparations with silver grains associated with the wedge-shaped $D f^{+}$ homologue.

Our analysis confirms that the hybridization site for $\lambda \mathrm{DmA} 2$ is $5 \mathrm{C}$. Furthermore, this site must reside within the region where $D f C 149$ and $D f N 73$ overlap since hybridization of the probe was restricted to one homologue of heterozygous deficient chromosomes for either $D f C 149$ or $D f N 73$. The distribution of the silver grains to the left end of the $D f^{+} N 73$ homologue (Fig. 2e) and at the right end of the $D f^{+} C 149$ homologue (Fig. 2b) also agrees with this conclusion. According to Bridges' (1938) map of the X chromosome the $5 \mathrm{C}$ region consists of 10 bands; Dr. G. Lefevre (personal communication) places the right break of $D f C 149$ at $5 \mathrm{C} 4 \pm$ and the left break of $D f N 73$ at $5 \mathrm{C} 3$. Thus, the position of the actin gene is narrowed to band $5 \mathrm{C} 3$ or $5 \mathrm{C} 4$.

This analysis does not, unfortunately, indicate coincidence between a known morphological lesion and the actin gene. We were originally encouraged to undertake this study by the fact that 3 of the 6 hybridization sites for actin genes are located at or near loci for crossveinless $(\mathrm{cv})$ mutants: $5 \mathrm{C}$ and $c v$ on the $\mathrm{X}$ chromosome; $57 \mathrm{~A}$ and $c v-2$ on the second chromosome; $87 \mathrm{E}$ and $c v-c$ on chromosome 3 . The $C 149$ chromosome is deficient for $c v$ whereas the $N 73$ chromosome is not. The actin gene is located within the overlap region of these two deficiencies, thus precluding a correlation between it and the $c v$ locus which lies within the region of the chromosome covered by the $C 149$ deficiency but outside the region of its overlap with $D f N 73$. On the other hand, the proximity of $c v$ to the hybridization site for $\lambda \mathrm{DmA} 2$ suggests that this mutant will be useful for screening deletions in the vicinity of the $5 \mathrm{C}$ actin gene. Direct analysis of the transcriptional activity at $5 \mathrm{C}$ as well as the kind and amount of actin found in the $c v$ mutant may provide a better assessment of a possible interaction between these two loci at $5 \mathrm{C}$.

The results presented here constitute an improvement of the in situ hybridization technique for the actin genes. We think that the use of a DNA probe with lower specific activity resulted in low backgrounds. Use of a DNA probe also reduced the amount of manipulation of the chromosome preparations since the post-hybridization RNase step was eliminated. Furthermore, the resolving power of in situ hybridization was increased by cytogenetic analysis so it was unnecessary to use giant larvae to obtain large chromosomes to visualize the hybridization site. Combination of cytogenetic and molecular genetic approaches will no doubt be useful for assigning DNA probes to specific bands of the salivary gland chromosomes.

Acknowledgements. We thank Dr. G. Lefevre for information on the deficiencies and Dr. D.B. Roberts for comments on the manuscript. This research was supported by a grant from the Muscular Dystrophy Association and NIH grant HD14145 to A.S. and NIH grant AG01945 to T.M.R.

\section{References}

Bonner, J.J., Pardue, M.L.: Ecdysone-stimulated RNA synthesis in imaginal discs of Drosophila melanogaster. Chromosoma (Berl.) 58, 87-89 (1976) 
Bridges, C.B.: A revised map of the salivary gland X-chromosome of Drosophila melanogaster. J. Heredity 29, 11-13 (1938)

Bridges, C.B., Bridges, P.N.: A new map of the second chromosome: A revised map of the right limb of the second chromosome of Drosophila melanogaster. J. Heredity 30, 475-476 (1939)

Bridges, P.N.: A revised map of the left limb of the third chromosome of Drosophila melanogaster. J. Heredity 32, 64-65 (1941)

Fyrberg, E.A., Bond, B.J., Hershey, N.D., Mixster, K.S., Davidson, N.: The actin genes of Drosophila: Protein coding regions are highly conserved, but intron positions are not. Cell 24, 107-116 (1981)

Fyrberg, E.A., Kindle, K.L., Davidson, N., Sodja, A.: The actin genes of Drosophila: A highly dispersed family. Cell 19, 365-378 (1980)

Gall, J., Pardue, M.L.: Nucleic acid hybridization in cytological preparations. In: Methods of Enzymology 21 (L. Grossman and K. Moldave, eds.), pp. 470-480. New York: Academic Press 1971

Huxley, H.E.: The mechanism of muscle contraction. Science 164, 1356-1366 (1969)

Korn, E.D.: Biochemistry of actomyosin-dependent cell motility (A review). Proc. Natl. Acad. Sci. (Wash.) 75, 588-599 (1978)

Lindsley, D.L., Grell, E.H.: Genetic variations of Drosophila melanogaster. Carnegie Inst. Wash. Publ. 627 (1968)

Maniatis, T., Jeffrey, A., Kleid, D.G.: Nucleotide sequence of the rightward operator of phage 2. Proc. Natl. Acad. Sci. (Wash.) 72, 1184-1188 (1975)

Pollard, T.D., Weihing, R.R.: Actin and myosin and cell movement. In: Critical Review in Biochemistry vol. 2 (G. Fasmon ed.), pp. 1-65. Cleveland, Ohio: CRC Press 1974

Sodja, A., Arking, R., Zafar, R.S.: Actin gene expression during embryogenesis of Drosophila melanogaster. Dev. Biol. 90, 363-368 (1982)

Vandekerckhove, J., Weber, K.: Mammalian cytoplasmic actins are the product of at least two genes and differ in primary structure in at least 25 identified positions from skeletal muscle actins. Proc. Natl. Acad. Sci. (Wash.) 75, 1106-1110 (1978a)

Vandekerckhove, J., Weber, K.: At least six different actins are expressed in a higher mammal: An analysis based on the amino acid sequence of the amino-terminal try-tic peptide. J. Mol. Biol. 126, 783-803 (1978b)

Received June 1, 1982 / Accepted by J.H. Taylor 